\title{
As plantas e seus usos nos quintais de Alta Floresta, Mato Grosso
}

\section{Plants and uses in Alta Floresta homegardens, Mato Grosso, Brazil}

\author{
Jociane Rosseto de Oliveira Silva ${ }^{1}{ }^{1}$; Ivan Cleiton de Oliveira Silva ${ }^{2}{ }^{2}$ Maria de Fatima Barbosa \\ Coelho $\mathbb{D}^{3}$; Elisangela Clarete Camili $\mathbb{D}^{4}$
}

${ }^{1}$ Mestre em Agricultura Tropical, Coordenação de Engenharia Florestal, Universidade do Estado de Mato Grosso, Campus II Perimetral Rogério Silva, Jardim Flamboyant, Alta Floresta, Mato Grosso, Brasil. E-mail: jorosseto@ hotmail.com; ${ }^{2}$ Mestre em Ciências Ambientais, Professor da Universidade do Estado de Mato Grosso, Campus II Perimetral Rogério Silva, Jardim Flamboyant, Alta Floresta, Mato Grosso, Brasil. E-mail: ivanunemataf@unemat.br; ${ }^{3}$ Doutora em Fitotecnia, Programa de Pós Graduação em Agricultura Tropical, Universidade Federal de Mato Grosso, Cuiabá, Mato Grosso, Brasil, E-mail: coelhomfstrela@gmail.com; ${ }^{4}$ Doutora em Agronomia (Horticultura), Professora da Universidade Federal de Mato Grosso, Cuiabá, Mato Grosso, Brasil, E-mail: eccamili@ hotmail.com.

\section{A R T I G O}

Recebido: 27/10/2018

Aprovado: 26/03/2019

\section{Palavras-chave:}

Plantas medicinais

Biodiversidade

Segurança alimentar

Key words:

Medicinal plant

Biodiversity

Food security

\section{R E S U M O}

As plantas dos quintais urbanos são utilizadas para múltiplas finalidades, com destaque para as espécies alimentícias e medicinais. $\mathrm{O}$ objetivo do presente estudo foi realizar o levantamento das espécies vegetais e seus respectivos usos nos quintais de dois bairros em Alta Floresta, estado de Mato Grosso e estabelecer o perfil dos informantes. Na seleção dos entrevistados aplicou-se a amostragem bola de neve, onde foram utilizadas as técnicas de observação direta e participante, sendo os principais envolvidos na manutenção dos quintais indagados sobre o manejo das plantas e as partes utilizadas para cada uso. Foram observados 99 táxons pertencentes a 49 famílias botânicas. As famílias mais frequentes foram Lamiaceae, Fabaceae e Myrtaceae. As espécies Cocos nucifera L., Myrciaria cauliflora (Mart.) O.Berg e Lippia alba (Mill.) N.E.Br. ex P. Wilson estavam presentes em $50 \%$ dos quintais. As categorias de uso citadas pelos informantes foram: a) arborização; b) condimentar; c) cultivo; d) frutífera; e) medicinal e f) paisagismo. Mais de $50 \%$ das espécies destina-se ao uso alimentício, destacando a função de segurança alimentar dos quintais. A segunda categoria com maior número de espécies foi a de uso medicinal com 29 táxons, pertencentes à 17 famílias e, Lamiaceae foi a família com maior número de espécies usadas. O uso medicinal mais citado foi na forma de decocção (chá) para gripes e resfriados.

\section{A B S T R A C T}

The plants of the urban homegardens are used for multiple purposes with emphasis on food and medicinal species. The aim of the present study was to survey the plant species and their respective uses in the homegardens of two neighborhoods in Alta Floresta, Mato Grosso State and to establish the profile of the informants. In the selection of the interviewees the snowball sampling was used where the techniques of direct observation and participant were used, being the main ones involved in the maintenance of the quintals inquired about the management of the plants and the parts used for each use. A total of 99 taxa belonging to 49 botanical families were observed. The most frequent families were Lamiaceae, Fabaceae and Myrtaceae. Cocos nucifera L., Myrciaria cauliflora (Mart.) O.Berg and Lippia alba (Mill.) N.E.Br. ex P. Wilson were the species present in 50\% of the homegardens. The categories of use cited by the informants were: a) afforestation; b) seasoning; c) cultivation; d) fruitful; e) medicinal and f) landscaping. More than $50 \%$ of the species is destined for food use, highlighting the food security function of the backyards. The second category with the greatest number of species was the one of medicinal use with 29 taxa belonging to 17 families, and Lamiaceae was the family with the largest number of species used. The most cited medicinal use was in the form of decoction (tea) for colds and flus.

\section{Revista Verde \\ ISSN 1981-8203 \\ Pombal, Paraíba, Brasil}

v. 14, n.3, jul.-set, p.420-428, 2019

doi: 10.18378/rvads.v14i3.6499 


\section{INTRODUÇÃO}

A área no entorno das residências, onde é cultivada uma mistura de espécies agrícolas e florestais e, onde são criados pequenos animais domésticos é denominada quintal (KUMAR; NAIR, 2006).

A manutenção da tradição em cultivar diferentes espécies no quintal está relacionada com a segurança alimentar e aumento da renda familiar (AMARAL et al., 2016), introdução e domesticação de espécies (GAO et al., 2012), eficiência do uso da terra, conservação de espécies e sustentabilidade (KUMAR; NAIR, 2006; FLORENTINO et al., 2007), aumento da biodiversidade (OAKLEY, 2004) e cuidados com a saúde. Os quintais são importantes na conservação de plantas medicinais por comunidades rurais e urbanas (BAJPAI et al., 2013; PERNA; LAMANO-FERREIRA, 2014).

Oakley (2004) em diversos estudos realizados na Ásia, África e América Latina confirma que os quintais domésticos contêm espécies de ciclo curto, contribuindo para alimentar as famílias durante o período da fome, até a colheita dos cultivos principais, que são reservas estratégicas de material genético, funcionam como espaços de conservação de variedades especiais ou preferenciais, e como locais de experimentação de novas variedades.

No Brasil destacam-se os usos das espécies vegetais mantidas nos quintais domésticos para fins alimentícios e medicinais (FLORENTINO et al., 2007; GUARIM NETO; AMARAL, 2010; AGUIAR; BARROS, 2012; FREITAS et al., 2012; EICHEMBERG; AMOROZO, 2013; FREITAS et al., 2015; GARCIA et al., 2015; PEREIRA; FIGUEIREDO NETO, 2015).

No município de Aripuanã região Norte de Mato Grosso, onde a vegetação é caracterizada como Floresta Amazônica, Brito (1996) relatou dentre as espécies identificadas nos quintais, os principais usos como sendo ornamental (102 espécies), alimentício (79 espécies) e medicinal (53 espécies).

Em Alta Floresta foram conduzidos dois estudos sobre a diversidade de plantas nos quintais. Em um deles, Santos (2004) verificou que os quintais são destinados ao manejo de árvores, arbustos e ervas, para diferentes finalidades, com espécies perenes e anuais, cultivadas em consórcio com pequenos animais, sendo a responsabilidade pela manutenção, exercida principalmente pelas mulheres, que complementam a renda familiar com a venda de alguns vegetais produzidos, especialmente as plantas olerícolas. Em outro estudo realizado em Alta Floresta, Gervazio (2015) observou 30 quintais, onde constatou-se que o maior número de espécies cultivadas eram das famílias Fabaceae, Asteraceae, Myrtaceae e Lamiaceae e o maior número de indivíduos eram das famílias Euphorbiaceae, Musaceae, Malvaceae e Poaceae, com uma média de 236,87 plantas por quintal.

Considerando que a região se encontra em processo de expansão demográfica e há necessidade de estudos sobre a composição florística dos quintais, o objetivo no presente trabalho foi realizar o levantamento das espécies vegetais e seus respectivos usos nos quintais de dois bairros em Alta Floresta/MT

\section{MATERIAL E MÉTODOS}

O município de Alta Floresta possui área de $8.947,07 \mathrm{~km}^{2}$ e situa-se na região norte do estado de Mato Grosso, a $830 \mathrm{~km}$ de distância da capital, Cuiabá. Alta Floresta foi fundada em 19 de maio de 1976 com famílias na maioria vindas do sul do Brasil para realizar atividades agrícolas. Está localizado nas coordenadas $09^{\circ} 52^{\prime} 32^{\prime \prime} \mathrm{S}$ e $56^{\circ} 05^{\prime} 10^{\prime \prime} \mathrm{W}$, com população de 49.233 habitantes, segundo o censo de 2010 (IBGE, 2010).

O clima é tropical chuvoso e, segundo Koppen é classificado como Am, com duas estações bem definidas, verão chuvoso e inverno seco e, apresenta elevado índice pluviométrico no verão, podendo atingir médias superiores a $2.750 \mathrm{~mm}$ com intensidade máxima em janeiro, fevereiro e março, com nítido período de seca. As temperaturas ficam entre 20 a $38{ }^{\circ} \mathrm{C}$, tendo em média $26{ }^{\circ} \mathrm{C}$, podendo chegar aos $40{ }^{\circ} \mathrm{C}$ nos dias mais quentes em alguns pontos do município. A altitude é de 250 a $450 \mathrm{~m}$ acima do nível do mar (ALVARES et al., 2014).

O presente estudo foi realizado em dezesseis quintais de dois Bairros de Alta Floresta/MT: Setor F (no centro) e Cidade Alta (na periferia). Na seleção dos entrevistados foi aplicada a técnica de amostragem bola de neve (snowball), ou seja, iniciou-se a entrevista com a pessoa responsável por um quintal, escolhida aleatoriamente, no final da entrevista solicitou-se que o entrevistado indicasse outra pessoa e assim, sucessivamente. Foram usadas as técnicas de observação direta e participante, onde os principais envolvidos na manutenção dos quintais são indagados sobre o manejo das plantas e as partes empregadas para cada uso e também sobre o perfil socioeconômico (idade, escolaridade, origem, tempo de residência, etc.) (ALBUQUERQUE et al., 2014).

Assim como citado em Albuquerque et al. (2014) realizouse a comprovação in loco das espécies citadas pelos informantes nas entrevistas.

Em cada quintal visitado foram feitos levantamentos das espécies vegetais com a coleta de amostras para exsicatas e identificação no Herbário da Universidade Federal de Mato Grosso. As informações foram compiladas sob a forma de um banco de dados, utilizando-se matrizes de textos, conforme metodologia proposta por Amorozo e Viertler (2010).

\section{RESULTADOS E DISCUSSÃO}

O município de Alta Floresta/MT foi formado com migrantes de outros Estados brasileiros. Neste estudo verificouse que a maioria dos entrevistados é procedente do estado do Paraná, seguido de Minas Gerais, Maranhão e Rondônia (Figura 1A). Segundo Guarim Neto e Novais (2008) isso reflete o processo de colonização da região norte de Mato Grosso devido à migração das pessoas oriundas da região sul do Brasil para áreas poucas exploradas, objetivando a compra de áreas maiores e produtivas por baixo custo.

Todos os proprietários dos quintais possuem algum grau de formação acadêmica como pode ser verificado na Figura 1B. A maioria cursou o ensino médio ou tem o terceiro grau incompleto, situação diferente da encontrada em outros estudos realizados no Mato Grosso e Rio Grande do Norte, onde 
verificou-se uma baixa escolaridade entre os proprietários (AMARAL; GUARIM NETO, 2008; CARNIELLO et al., 2010; FREITAS et al., 2012; FREITAS et al., 2015).

Figura 1. Estado de origem (A) e grau de escolaridade (B) dos entrevistados em Alta Floresta, Mato Grosso, Brasil.

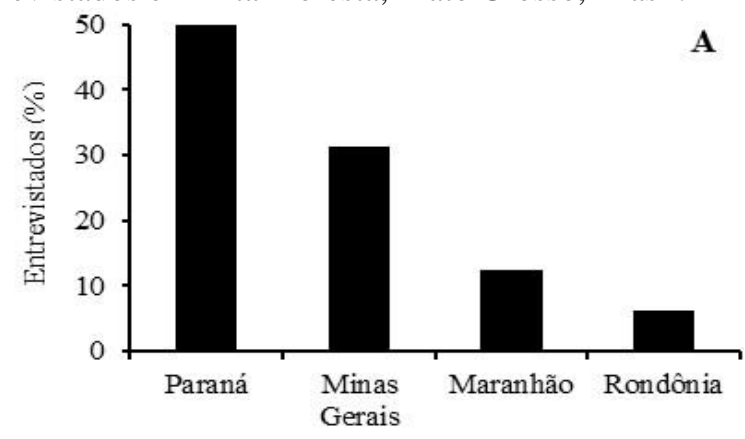

Estado de Origem dos Entrevistados

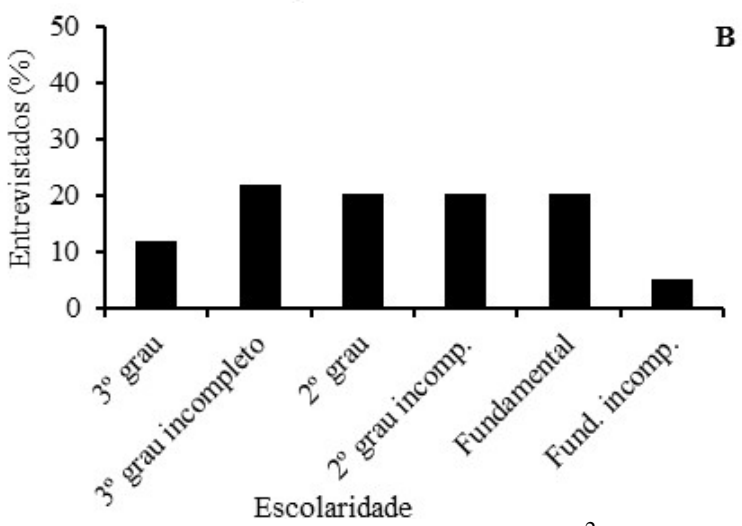

Os quintais apresentaram em média $703 \mathrm{~m}^{2}$ de área total (Figura 2A). Os lotes em Alta Floresta são relativamente grandes, como ocorre em outros municípios do Estado. Em estudo realizado em Mirassol do Oeste/MT, constatou-se que a extensão territorial de $97 \%$ dos lotes variou entre 200 a $1000 \mathrm{~m}^{2}$ (CARNIELLO et al., 2010). Em Rosário Oeste/MT a área média dos quintais analisados foi de $622 \mathrm{~m}^{2}$, incluindo a área do domicílio, sendo que todos os terrenos possuíam o formato retangular (AMARAL; GUARIM NETO, 2008). Em outro estudo, conduzido por Gervazio (2015) em Alta Floresta observou-se que os quintais possuíam área média de $2.500 \mathrm{~m}^{2}$.

Dos entrevistados, $43,75 \%$ responderam que residem no local entre 1 a 5 anos, período relativamente curto de tempo para que um grande número de espécies seja cultivado (Figura 2B), no entanto, o tempo médio de residência nos quintais estudados é de 10,63 anos.

Verificou-se que $57 \%$ dos entrevistados são mulheres, as quais são responsáveis pela escolha das espécies a serem cultivadas, pelo plantio e manutenção dos quintais. Havendo, portanto, um equilíbrio em relação ao gênero na execução de tarefas nos quintais, resultado semelhante ao constatado por Santos et al. (2013) em quintais agroecológicos na comunidade Mem de Sá, Itaporanga d'Ajuda-SE; entretanto, esses resultados diferem da maioria dos estudos, onde observou-se predominância das mulheres como responsáveis pelos quintais
(FLORENTINO et al., 2007; FREITAS et al., 2012; AMARAL et al., 2016).

Figura 2. Área dos quintais (A) e tempo de residência (B) citados pelos entrevistados em Alta Floresta, Mato Grosso, Brasil.
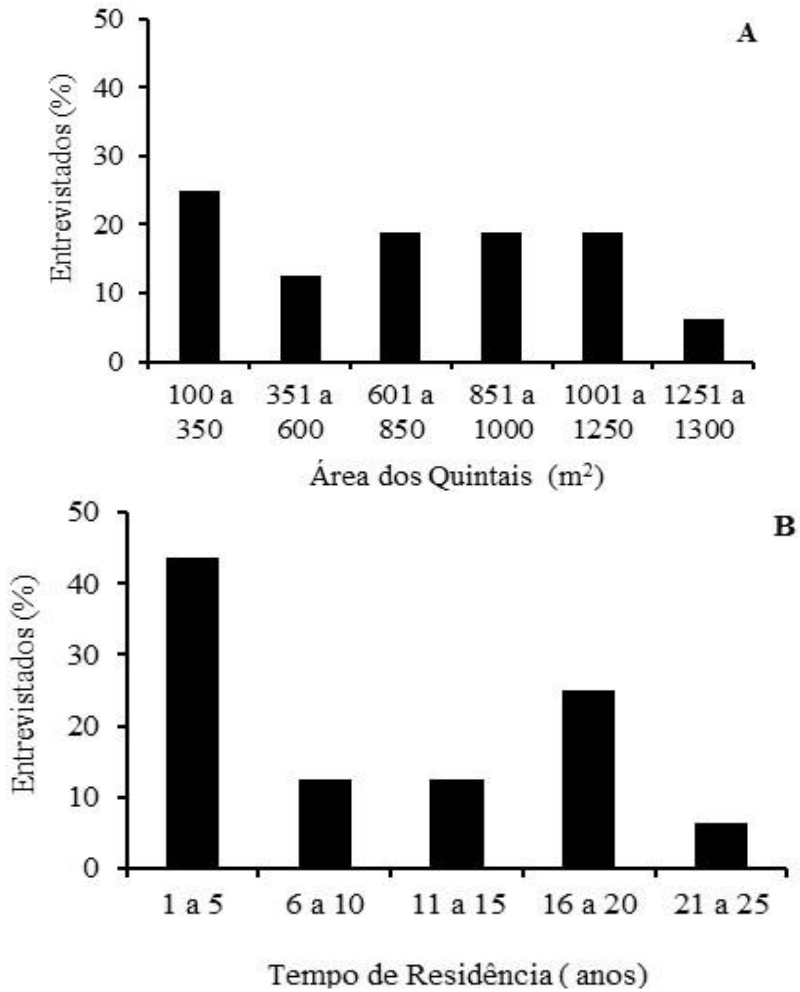

A composição florística observada nos quintais de Alta Floresta corresponde a 99 táxons pertencentes à 49 famílias botânicas (Tabela 1). As famílias mais frequentes foram Lamiaceae, Fabaceae e Myrtaceae (Figura 3). As espécies mais frequentes foram Cocos nucifera L., Myrciaria cauliflora (Mart.) O.Berg e Lippia alba (Mill.) N.E.Br. ex P. Wilson, presentes em $50 \%$ dos quintais. $C$. nucifera está presente com frequência em muitos quintais brasileiros (FREITAS et al., 2012; SANTOS et al., 2013; FREITAS et al., 2015), possivelmente por produzir frutos que podem ser comercializados para aumentar a renda das famílias e pelos múltiplos usos na alimentação e saúde. A presença de $M$. cauliflora pode estar relacionada com a origem dos entrevistados que na maioria são do Paraná, pois, a espécie é originária da Mata Atlântica.

O hábito de crescimento predominante foi o herbáceo, com 50 espécies, enquanto o arbustivo e arbóreo foram representados por 25 e 24 espécies, respectivamente. A predominância de espécies herbáceas foi observada em outros estudos (PILLA et al., 2006; ALBERTASSE et al., 2010), possivelmente pela- facilidade de se cultivar ervas em quintais (PILLA et al., 2006). 
Tabela 1. Plantas encontradas nos quintais com suas respectivas famílias, nomes vernaculares, hábito de crescimento, categoria de uso, origem ( $\mathrm{N}$ - nativa ou $\mathrm{E}$ - exótica) e frequência de quintais em que estão presentes em dois bairros de Alta Floresta, Mato Grosso, Brasil.

\begin{tabular}{|c|c|c|c|c|c|}
\hline Família/espécie & Nome vernacular & $\begin{array}{l}\text { Hábito de } \\
\text { crescimento }\end{array}$ & $\begin{array}{l}\text { Categoria de } \\
\text { uso }\end{array}$ & Origem & Frequência \\
\hline \multicolumn{6}{|l|}{ Aliaceae } \\
\hline $\begin{array}{l}\text { Allium fistulosum L. } \\
\text { Alismataceae }\end{array}$ & cebolinha & herbáceo & condimentar & $\mathrm{E}$ & 37,5 \\
\hline $\begin{array}{l}\text { Echinodorus grandiflorus (Cham. \& Schltr.) } \\
\text { Micheli }\end{array}$ & chapéu-de-couro & herbáceo & medicinal & $\mathrm{N}$ & 6,3 \\
\hline \multicolumn{6}{|l|}{ Amaranthaceae } \\
\hline Alternanthera brasiliana (L.) Kuntze & terramicina & herbáceo & medicinal & $\mathrm{N}$ & 25,0 \\
\hline \multicolumn{6}{|l|}{ Anacardiaceae } \\
\hline Anacardium occidentale L. & caju & arbóreo & frutífera & $\mathrm{N}$ & 18,8 \\
\hline $\begin{array}{l}\text { Mangifera indica } \mathrm{L} \text {. } \\
\text { Annonaceae }\end{array}$ & \multicolumn{4}{|c|}{ Annonaceae } & 18,8 \\
\hline $\begin{array}{l}\text { Annona squamosa L. } \\
\text { Apiaceae }\end{array}$ & atta, pinha & arbustivo & frutífera & $\mathrm{E}$ & 18,8 \\
\hline \multicolumn{6}{|l|}{ Araceae } \\
\hline Dieffenbachia picta Schott & $\begin{array}{l}\text { comigo-ninguém- } \\
\text { pode }\end{array}$ & herbáceo & paisagismo & $\mathrm{E}$ & 18,8 \\
\hline \multicolumn{6}{|l|}{ Arecaceae } \\
\hline Cocos nucifera $\mathrm{L}$. & coco, coco-verde & arbóreo & frutífera & $\mathrm{E}$ & 50,0 \\
\hline $\begin{array}{l}\text { Acrocomia aculeata (Jacq.) Lodd. ex Mart } \\
\text { Aristolochiaceae }\end{array}$ & bocaiuva, macaúba & arbóreo & frutífera & $\mathrm{N}$ & 18,8 \\
\hline $\begin{array}{l}\text { Aristolochia esperanzae Kuntze } \\
\text { Asteraceae }\end{array}$ & cipó-mil-homens & herbáceo & medicinal & $\mathrm{N}$ & 18,8 \\
\hline Lactuca sativa $\mathrm{L}$. & alface & herbáceo & cultivo & $\mathrm{E}$ & 18,8 \\
\hline Mikania glomerata Spreng. & guaco & herbáceo & medicinal & $\mathrm{N}$ & 12,5 \\
\hline Cichorium intybus L. & chicória & herbáceo & cultivo & $\mathrm{E}$ & 12,5 \\
\hline \multicolumn{5}{|l|}{ Betulaceae } & 6,3 \\
\hline \multicolumn{5}{|l|}{ Bignoniaceae } & 12,5 \\
\hline Tecoma stans (L.) Juss ex. Kenth. & ipê-de-jardim & arbustivo & arborização & $\mathrm{E}$ & 6,3 \\
\hline \multicolumn{6}{|l|}{ Boraginaceae } \\
\hline \multicolumn{6}{|l|}{ Brassicaceae } \\
\hline Brassica oleracea var. acephala L. & couve-de-folhas & herbáceo & cultivo & $\mathrm{E}$ & 12,5 \\
\hline Eruca sativa (Mill.) & rúcula & herbáceo & cultivo & $\mathrm{E}$ & 12,5 \\
\hline \multicolumn{6}{|l|}{ Bromeliaceae } \\
\hline Aechmea blanchetiana (Baker) L.B.Sm. & bromélia & herbáceo & paisagismo & $\mathrm{N}$ & 6,3 \\
\hline \multicolumn{5}{|l|}{ Cariacaceae } & 6,3 \\
\hline $\begin{array}{l}\text { Carica papaya } \mathrm{L} . \\
\text { Celastraceae }\end{array}$ & mamão & arbustivo & frutífera & $\mathrm{E}$ & 37,5 \\
\hline $\begin{array}{l}\text { Maytenus ilicifolia (Schrad.) Planch. } \\
\text { Chrysobalanaceae }\end{array}$ & espinheira-santa & arbustivo & medicinal & $\mathrm{N}$ & 12,5 \\
\hline $\begin{array}{l}\text { Licania tomentosa Benth. Fritsch } \\
\text { Costaceae }\end{array}$ & oiti & arbóreo & arborização & $\mathrm{N}$ & 12,5 \\
\hline $\begin{array}{l}\text { Costus spiralis (Jacq.) Roscoe } \\
\text { Cupressaceae }\end{array}$ & caninha-do-brejo & herbáceo & medicinal & $\mathrm{N}$ & 25,0 \\
\hline $\begin{array}{l}\text { Chamaecyparis lawsoniana (A.Murray) Parl. } \\
\text { Cucurbitaceae }\end{array}$ & pinheiro & arbóreo & arborização & $\mathrm{E}$ & 18,8 \\
\hline Cucurbita moschata Duchesne & abóbora & herbáceo & cultivo & $\mathrm{E}$ & 12,5 \\
\hline
\end{tabular}




\section{Euphorbiaceae}

Manihot esculenta Crantz

Codiaeum variegatum $\mathrm{L}$.

Fabaceae

Enterolobium maximum Ducke

Hymenolobium excelsum Ducke

Parkia multijuga Benth.

Hymenaea courbaril L.

Torresea acreana Ducke

Phaseolus vulgaris L.

Heliconiaceae

Heliconia chartacea Lane ex Barreiros

Lamiaceae

Plectranthus barbatus Andr.

Plectranthus amboinicus (Lour.) Spreng.

Ocimum micranthum Willd.

Mentha pulegium L.

Mentha piperita $\mathrm{L}$.

Ocimum basilicum $\mathrm{L}$.

Leonurus sibiricus L.

Melissa officinalis L.

Origanum vulgare L.

Rosmarinus officinalis L.

Lauraceae

Persea americana Mill.

Liliaceae

Sansevieria trifasciata Hort.

Aloe vera $\mathrm{L}$. Burm.

Malphiguiaceae

Malpighia glabra L.

Malvaceae

Ceiba pentranda (L.) Gaertn.

Gossypium barbadense L.

Hibiscus rosa-sinensis L.

Malva sylvestris L.

Theobroma grandiflorum (Willd. ex Spreng.)

K.Schum.

Theobroma cacao L.

Meliaceae

Azadirachta indica A.Juss.

Swietenia macrophylla King

Moraceae

Ficus benjamina L.

Artocarpus integrifolia $\mathrm{L}$.

Morus nigra L.

Musaceae

Musa sp.

Myrtaceae

Psidium guajava L.

Eugenia uniflora L.

Myrciaria cauliflora (Mart.) O.Berg

Syzygium cumini (L.) Skeels

Eugenia stipitata McVaugh

Eugenia uvalha Cambess.

Nyctaginaceae

Bougainvillea spectabilis Willd.

Orchidaceae

Cattleya spp.

Oxalidaceae

\begin{tabular}{|c|c|c|c|c|}
\hline mandioca & arbustivo & cultivo & $\mathrm{N}$ & 12,5 \\
\hline cróton & arbustivo & paisagismo & $\mathrm{E}$ & 6,3 \\
\hline timburil & arbóreo & arborização & $\mathrm{N}$ & 6,3 \\
\hline angelim & arbóreo & arborização & $\mathrm{N}$ & 6,3 \\
\hline barjão & arbóreo & arborização & $\mathrm{N}$ & 6,3 \\
\hline jatobá & arbóreo & paisagismo & $\mathrm{N}$ & 12,5 \\
\hline cerejeira & arbóreo & arborização & $\mathrm{N}$ & 6,3 \\
\hline feijão & herbáceo & cultivo & $\mathrm{E}$ & 12,5 \\
\hline helicônia & herbáceo & paisagismo & $\mathrm{N}$ & 12,5 \\
\hline boldo & herbáceo & medicinal & $\mathrm{E}$ & 43,7 \\
\hline malvarisco & herbáceo & medicinal & $\mathrm{E}$ & 25,0 \\
\hline alfavaca & herbáceo & medicinal & $\mathrm{E}$ & 31,3 \\
\hline poejo & herbáceo & medicinal & $\mathrm{E}$ & 31,3 \\
\hline menta & herbáceo & medicinal & $\mathrm{E}$ & 18,8 \\
\hline manjericão & herbáceo & medicinal & $\mathrm{E}$ & 12,5 \\
\hline macaé & herbáceo & medicinal & $\mathrm{E}$ & 6,3 \\
\hline cidreira & herbáceo & medicinal & $\mathrm{E}$ & 18,8 \\
\hline orégano & herbáceo & condimentar & $\mathrm{E}$ & 6,3 \\
\hline alecrim & herbáceo & medicinal & $\mathrm{E}$ & 25,0 \\
\hline abacate & arbóreo & frutífera & $\mathrm{E}$ & 6,3 \\
\hline espada-de-São-Jorge & herbáceo & paisagismo & $\mathrm{E}$ & 12,5 \\
\hline babosa & herbáceo & medicinal & $\mathrm{E}$ & 6,3 \\
\hline acerola & arbustivo & frutífera & $\mathrm{E}$ & 37,5 \\
\hline paineira & arbóreo & arborização & $\mathrm{N}$ & 6,3 \\
\hline algodão & arbustivo & medicinal & $\mathrm{E}$ & 25,0 \\
\hline hibisco & arbustivo & paisagismo & $\mathrm{E}$ & 12,5 \\
\hline malva & herbáceo & medicinal & $\mathrm{E}$ & 18,8 \\
\hline cupuaçu & arbustivo & frutífera & $\mathrm{N}$ & 25,0 \\
\hline cacau & arbustivo & frutífera & $\mathrm{E}$ & 12,5 \\
\hline $\operatorname{nim}$ & arbóreo & arborização & $\mathrm{E}$ & 6,3 \\
\hline mogno & arbóreo & arborização & $\mathrm{N}$ & 6,3 \\
\hline figueira & arbóreo & arborização & $\mathrm{E}$ & 18,8 \\
\hline jaca & arbóreo & frutífera & $\mathrm{E}$ & 18,8 \\
\hline amora & arbóreo & frutífera & $\mathrm{E}$ & 25,0 \\
\hline banana & arbóreo & frutífera & $\mathrm{E}$ & 37,5 \\
\hline goiaba & arbustivo & frutífera & $\mathrm{E}$ & 25,0 \\
\hline pitanga & arbustivo & frutífera & $\mathrm{N}$ & 12,5 \\
\hline jabuticaba & arbustivo & frutífera & $\mathrm{N}$ & 50,0 \\
\hline jambolão & arbóreo & frutífera & $\mathrm{E}$ & 12,5 \\
\hline araçá-boi & arbóreo & frutífera & $\mathrm{N}$ & 6,3 \\
\hline uvaia & arbóreo & frutífera & $\mathrm{N}$ & 18,8 \\
\hline primavera & arbustivo & paisagismo & $\mathrm{N}$ & 25,0 \\
\hline orquídea & herbáceo & paisagismo & $\mathrm{N}$ & 31,3 \\
\hline
\end{tabular}

Revista Verde, v.14, n.3, p420-428, 2019 
Averrhoa carambola L.

Papaveraceae

Chelidonium majus L.

Passifloraceae

Passiflora edulis Sims

\section{Poaceae}

Saccharum officinarum L.

Bambusa gracilis Hort. ex Rivière \& C. Rivière

Cymbopogon citratus (DC) Stapf.

Polypodiaceae

Polypodium lepidopteris (Langds. \& Fisch.)

Kunze

Portulacaceae

Portulaca grandiflora Hook.

Punicaceae

Punica granatum L.

Rubiaceae

Mussaenda erythrophylla Schumach \& Thonn.

Ixora chinensis Lam.

\section{Rutaceae}

Citrus $x$ aurantium L.

Citrus $x$ limon (L.) Osbeck

Citrus $x$ sinensis (L.) Osbeck

Pilocarpus microphyllus Stapf ex Wardlew.

Ruta graveolens L.

Hydrangeaceae

Hydrangea macrophylla (Thunb.) Ser.

Solanaceae

Capsicum spp.

Solanum lycopersicum L.

Capsicum annuum $\mathrm{L}$.

\section{Verbenaceae}

Duranta repens L.

Lippia alba (Mill.) N.E.Br. ex P. Wilson

\section{Vitaceae}

Vitis vinifera L.

Zingiberaceae

Zingiber officinale Roscoe

Alpinia zerumbet (Pers.) B.L.Burtt \& R.M.Sm.

Curcuma aromatica Salisb. carambola

figatil

maracujá

cana-de-açúcar

bambu-de-jardim

capim-santo

samambaia

onze-horas

romã

mussaenda

ixora

Laranja-azeda

limão

laranja

jaborandi

arruda

hortência

pimentas

tomate

pimentão

pingo-de-ouro

cidreira

uva

gengibre

colônia

açafrão arbóreo

frutífera

herbáceo

medicinal

herbáceo

frutífera

arbustivo

cultivos

herbáceo

herbáceo

paisagismo

medicinal

herbáceo

paisagismo

E

herbáceo

paisagismo

E

arbustivo

medicinal

E

31,3

arbustivo

paisagismo

paisagismo

E

E

18,8

arbustivo

18,8 frutífera

arbustivo

arbustivo

frutífera

frutífera

arbustivo

medicinal

herbáceo

medicinal

herbáceo

paisagismo

herbáceo

herbáceo

herbáceo

condimentar

cultivo

cultivo

arbustivo

paisagismo

medicinal

$\mathrm{N}$

$\mathrm{N}$

$\mathrm{N}$

$\mathrm{N}$

$\mathrm{N}$

arbustivo

frutífera

E

6,3

herbáceo

herbáceo

condimentar

medicinal

condimentar

\section{1,3}

31,3$$
\begin{aligned}
& 18,8 \\
& 12,5
\end{aligned}
$$$$
25,0
$$

arbusto

herbáceo

Figura 3. Famílias botânicas mais frequentes (A) e número de espécies (B) nos quintais de Alta Floresta, Mato Grosso, Brasil.

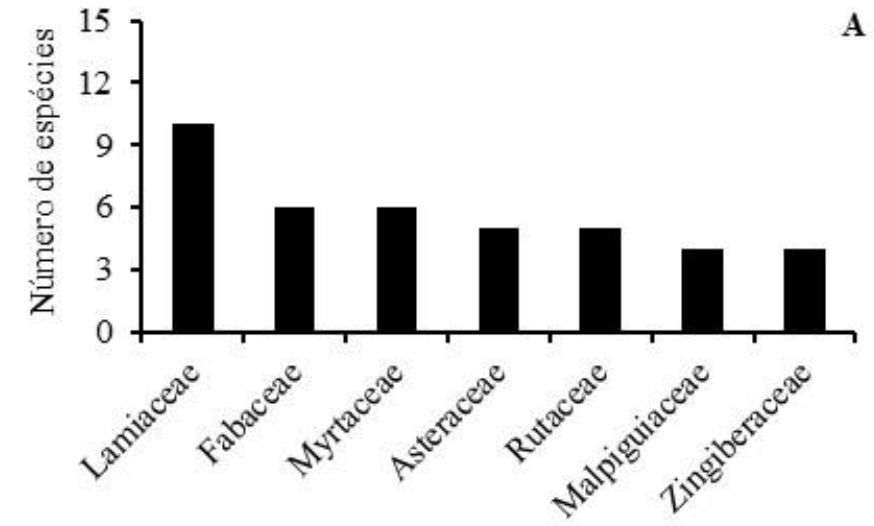

Famílias Botânicas

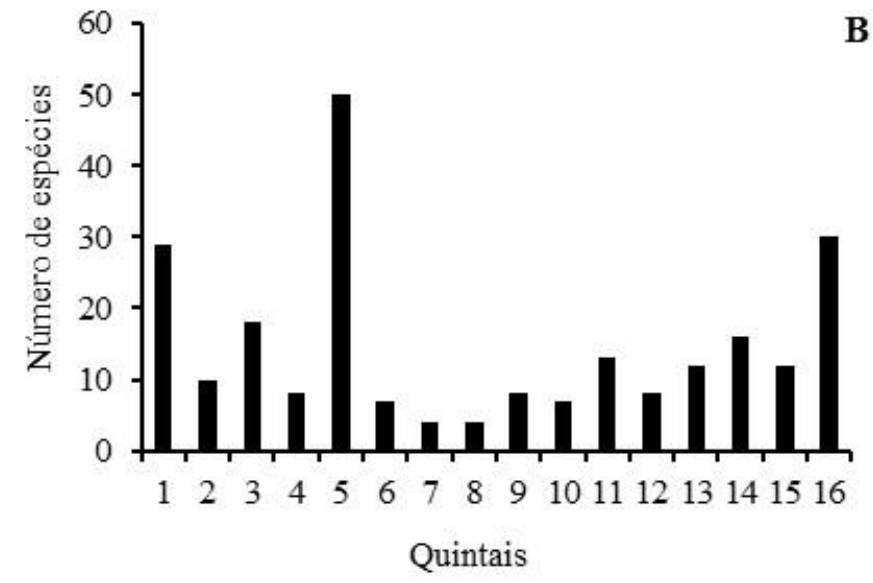

Revista Verde, v.14, n.3, p.420-428, 2019 
As categorias de uso citadas pelos informantes foram: a) arborização: espécies que proporcionam sombra, resultando em um ambiente agradável nos quintais; b) condimentar: espécies usadas no preparo de alimentos como tempero; c) cultivo: espécies herbáceas plantadas no quintal para alimentação; d) frutífera: espécies arbóreas que produzem frutos comestíveis; e) medicinal: espécies usadas para preparo de remédios caseiros e; f) paisagismo: espécies destinadas à ornamentação, decoração e embelezamento dos quintais (Figura 4).

A categoria de uso com maior número de espécies foi a medicinal, com 29 táxons, pertencentes à 17 famílias, com predominância da família Lamiaceae (Tabela 2). O uso mais frequente é na forma de chás para gripes e resfriados.
Figura 4. Categorias de uso das espécies de plantas presentes nos quintais de Alta Floresta, Mato Grosso, Brasil.

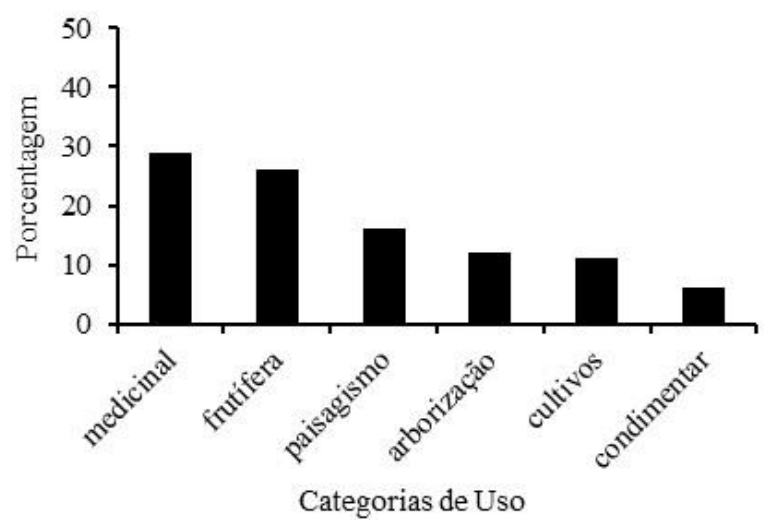

Tabela 2. Plantas medicinais identificadas nos quintais de Alta Floresta com seus respectivos usos. Alta Floresta, Mato Grosso, Brasil.

\begin{tabular}{|c|c|c|}
\hline Família/espécie & Nome vernacular & Usos \\
\hline \multicolumn{3}{|l|}{ Alismataceae } \\
\hline Echinodorus grandiflorus (Cham. \& Schltr.) & chapéu-de-couro & chá para o fígado e rins \\
\hline \multicolumn{3}{|l|}{ Micheli } \\
\hline \multicolumn{3}{|l|}{ Amaranthaceae } \\
\hline Alternanthera brasiliana (L.) Kuntze & terramicina & chá das folhas para dor, febre e infecções \\
\hline Chenopodium ambrosioides $\mathrm{L}$. & $\begin{array}{l}\text { erva-de-Santa- } \\
\text { Maria }\end{array}$ & sumo das folhas com leite para verminoses \\
\hline \multicolumn{3}{|l|}{ Aristolochiaceae } \\
\hline Aristolochia esperanzae Kuntze & cipó-mil-homens & estômago, fígado, raiz contra picada de cobra \\
\hline \multicolumn{3}{|l|}{ Asteraceae } \\
\hline Mikania glomerata Spreng. & guaco & xarope das folhas para gripe \\
\hline Calendula officinalis L. & calêndula & chá das flores para cicatrizar ferimentos \\
\hline \multicolumn{3}{|l|}{ Betulaceae } \\
\hline Corylus avellana $\mathrm{L}$. & avelã & cataplasma com a casca para cicatrizar úlceras \\
\hline \multicolumn{3}{|l|}{ Boraginaceae } \\
\hline Symphytum officinale L. & confrei & amassar as folhas para cicatrizar ferimentos \\
\hline \multicolumn{3}{|l|}{ Celastraceae } \\
\hline Maytenus ilicifolia (Schrad.) Planch. & espinheira-santa & chá das folhas para úlcera no estômago \\
\hline \multicolumn{3}{|l|}{ Costaceae } \\
\hline Costus spiralis (Jacq.) Roscoe & caninha-do-brejo & chá para os rins e pedra nos rins \\
\hline \multicolumn{3}{|l|}{ Lamiaceae } \\
\hline Plectranthus barbatus Andr. & boldo & chá para indigestão, dor no estômago e fígado \\
\hline Plectranthus amboinicus (Lour.) Spreng. & malvarisco & chá e lambedor para gripe e dor na garganta \\
\hline Ocimum micranthum Willd. & alfavaca & chá para gripe \\
\hline Mentha pulegium L. & poejo & xarope para gripe \\
\hline Mentha piperita $\mathrm{L}$. & menta & xarope para gripe \\
\hline Ocimum basilicum $\mathrm{L}$. & manjericão & xarope para gripe \\
\hline Leonurus sibiricus L. & macaé & chá para os rins \\
\hline Melissa officinalis L. & cidreira & chá das folhas como calmante \\
\hline Rosmarinus officinalis L. & alecrim & chá das folhas como estimulante \\
\hline \multicolumn{3}{|l|}{ Liliaceae } \\
\hline Aloe vera $\mathrm{L}$. Burm. & babosa & gel das folhas para queimaduras \\
\hline \multicolumn{3}{|l|}{ Malvaceae } \\
\hline Gossypium barbadense L. & algodão & chá das folhas usado no banho após o parto \\
\hline Malva sylvestris L. & malva & chá das folhas como anti-inflamatório \\
\hline \multicolumn{3}{|l|}{ Papaveraceae } \\
\hline Chelidonium majus L. & figatil & chá para problemas do fígado \\
\hline \multicolumn{3}{|l|}{ Poaceae } \\
\hline Cymbopogon citratus (DC) Stapf. & capim-santo & chá das folhas como calmante \\
\hline
\end{tabular}


Punica granatum $\mathrm{L}$.

Rutaceae

Pilocarpus microphyllus Stapf ex Wardlew.

Ruta graveolens $\mathrm{L}$.

Verbenaceae

Lippia alba (Mill.) N.E.Br. ex P. Wilson

Zingiberaceae

Alpinia zerumbet (Pers.) B.L.Burtt \& R.M.Sm. romã

chá da casca do fruto para a garganta e rouquidão

jaborandi arruda

cidreira colônia chá para problemas com os rins e para os cabelos chá das folhas para lavar os olhos

chá das folhas como calmante
Outros estudos etnobotânicos verificaram maior número de espécies de uso medicinal nas famílias Lamiaceae e Asteraceae, como constatado por Albertasse et al. (2010) no estado do Espírito Santo, Giraldi e Hanazaki (2010) em Santa Catarina, Pilla et al. (2006) em São Paulo e Moreira e Guarim Neto (2015) no Mato Grosso.

As espécies da família Lamiaceae destacam-se por serem ricas em óleos essenciais, o que confere às mesmas propriedades aromáticas e medicinais, daí a maior representatividade desta família neste e em diversos outros trabalhos sobre o levantamento das espécies vegetais em quintais domésticos (Mosca; Loiola, 2009; Freitas et al., 2012; Paulino et al., 2012; Alves; Povh, 2013).

Se forem agrupadas as espécies usadas na alimentação, incluindo as frutíferas, de cultivo e condimentares tem-se 42 espécies, ou seja, $42 \%$ do total informado, o que indica a importância dos quintais para a segurança alimentar das famílias. As frutas e hortaliças constituem valiosas fontes de nutrientes para as famílias, uma alternativa econômica para o consumo destes produtos em épocas de crise, e que, de outra maneira, seria de difícil obtenção (VALADÃO et al., 2006). Garcia et al. (2015) afirmam que os quintais exercem papel importante na segurança alimentar dos agricultores familiares, pela riqueza de espécies encontradas nos extratos arbóreos e arbustivos, em sua maioria por espécies que proporcionam alimentação saudável, com riqueza de nutrientes.

Em Boa Vista/RR, Semedo e Barbosa (2007) verificaram que o cultivo de árvores frutíferas em quintais caseiros segue um padrão que concentra a escolha em poucas espécies, nãooriginárias da Amazônia, mas, tradicionalmente consagradas pelo êxito na produção de frutos. No presente estudo a preferência por fruteiras exóticas também foi verificada, com destaque para a bananeira (Musa sp.). Nos quintais de Santarém/PA, Winklerprins (2010) constatou a presença de 33 espécies de plantas frutíferas em um total de 98 espécies.

$\mathrm{Na}$ categoria de arborização e paisagismo, existem muitas espécies que são plantadas com o objetivo de gerar sombra para os proprietários e, de acordo com os entrevistados, este espaço normalmente é o local onde os adultos conversam e descansam, as crianças brincam e realizam-se festas. As árvores também servem de abrigo para aves que vivem em áreas urbanas ou que estejam de passagem pelo local, e podem produzir frutos que eventualmente são consumidos por alguns animais frugívoros.

Nota-se ainda, a presença de espécies arbóreas trazidas da floresta Amazônica como Hymenolobium excelsum Ducke, Parkia multijuga Benth., Hymenaea courbaril L., Torresea acreana Ducke e Swietenia macrophylla King. O uso de espécies nativas do bioma da região é importante na conservação e domesticação e, Freitas et al. (2012) também constataram o uso de espécies nativas regionais nos quintais domésticos. Segundo Florentino et al. (2007) é comum observar a presença de espécies nativas nos quintais, que em geral estão relacionadas ao uso madeireiro, no entanto, em Alta Floresta são importantes para amenizar o calor e embelezar as ruas.

A maioria das espécies não são nativas (68 táxons), assim como constatado no levantamento feito em quintais de CáceresMT por Pereira e Figueiredo Neto (2015). Embora seja comum observar a presença de espécies nativas nos quintais das regiões tropicais úmidas e áridas, em todos há um domínio de plantas exóticas (ALBUQUERQUE et al., 2005). Embora não sejam nativas, são espécies cultivadas e utilizadas há muito tempo pela população brasileira, como é o caso dos representantes da família Lamiaceae (boldo, malvarisco, alfavaca, poejo, menta, manjericão, macaé, cidreira e alecrim).

Com relação à origem das espécies mantidas nos quintais, a maioria (82\%) foi obtida com amigos, as demais foram oriundas de vizinhos da zona rural, viveiro municipal do estado do Paraná, da cidade de Lucas do Rio Verde/MT, coletadas na Floresta e no Cerrado e adquiridas na feira do produtor. Esta situação difere do constatado por Pereira e Figueiredo Neto (2015) em Cáceres-MT, onde 37,98\% das plantas foram obtidas a partir de "mudas compradas" e as outras origens foram $18,98 \%$ de "mudas ganhas" e 24,06\% "encontradas no local".

\section{CONCLUSÕES}

Os quintais domésticos de Alta Floresta, Mato Grosso tem grande diversidade vegetal, cerca de 100 espécies pertencentes a 49 famílias botânicas.

As famílias mais frequentes são Lamiaceae, Fabaceae e Myrtaceae, com destaque para as espécies Cocos nucifera L., Myrciaria cauliflora (Mart.) O.Berg e Lippia alba (Mill.) N.E.Br. ex P. Wilson.

O uso alimentício seguido do emprego medicinal da maioria das espécies, indica a importância do incentivo à manutenção da diversidade nos quintais e dessas espécies para a segurança alimentar.

\section{AGRADECIMENTO}

Ao Conselho Nacional de Desenvolvimento Científico e Tecnológico $(\mathrm{CNPq})$ pela Bolsa de Produtividade concedida à segunda autora.

\section{REFERÊNCIAS}

AGUIAR, L. C. G. G.; BARROS, R. F. M. Plantas medicinais cultivadas em quintais de comunidades rurais no domínio do cerrado piauiense (Município de Demerval Lobão, Piauí, Brasil). Revista Brasileira de Plantas Medicinais, v. 14, n. 3, p. 419-434. 2012. 
ALBERTASSE, P. D.; THOMAZ, L. D.; ANDRADE, M. A. Plantas medicinais e seus usos na comunidade da Barra do Jucu, Vila Velha, ES. Revista Brasileira de Plantas Medicinais, v. 12, n. 3, p. 250-260, 2010.

ALBUQUERQUE, U. P.; ANDRADE, L. H. C.; CABALLERO, J. Structure and floristic of homegardens in Northeast of Brazil. Journal of Arid Environments, v. 62, p. 491-506, 2005.

ALBUQUERQUE, U. P.; CUNHA, L. V. F. C.; LUCENA, R. F. P.; ALVES, R. R. N. (Eds.) Methods and Techniques in Ethnobiology and Ethnoecology, Springer Protocols Handbooks, 2014. 480p.

ALVARES, C. A.; STAPE, J. L.; SENTELHAS, P. C.; GONÇALVES, J. L. M.; PAROVEK, G. Köppen's climate classification map for Brazil. Meteorologische Zeitschrift, Stuttgart, v. 22, n. 6, p. 711-728. 2014.

ALVES, G. S. P.; POVH, J. A. Estudo etnobotânico de plantas medicinais na comunidade de Santa Rita, Ituiutaba - MG. Biotemas, v. 26, n. 3, p. 231-242, 2013.

AMARAL C. N.; COELHO-DE-SOUZA, G. P.; SCHUCH, I.; SOUZA, M. Contribuições da produção de autoconsumo em quintais para a segurança alimentar e nutricional e renda em Jangada, Baixada Cuiabana, MT. Guaju, v. 2, n. 1, p. 102-119, 2016.

AMARAL, C. N.; NETO, G. G. Os quintais como espaços de conservação e cultivo de alimentos: um estudo na cidade de Rosário Oeste (Mato Grosso, Brasil). Boletim do Museu Paraense Emílio Goeldi. Ciências Humanas, v. 3, p. 329-341, 2008.

AMOROZO, M. C. M.; VIERTLER, R. B. A abordagem qualitativa na coleta e análise de dados em etnobiologia e etnoecologia. In: ALBUQUERQUE, U. P.; LUCENA, R. F. P.; CUNHA, L. V. F. C. Métodos e técnicas na pesquisa etnobiológica e etnoecológica. 3ed. Recife: NUPEEA, p. 6782, 2010.

BAJPAI, S.; SHARMA, A. K.; KANUNGO, V. K. Traditional home gardens: A preserve of medicinal plants. International Journal of Herbal Medicine, v. 1, n. 2, p. 152-161, 2013.

BRITO, M. A. Uso social da biodiversidade em quintais agroflorestais de Aripuanã-MT. 1996, 108f Dissertação (Programa de Pós-graduação em Ecologia e Conservação da Biodiversidade). Universidade Federal de Mato Grosso, Cuiabá. 1996.

CARNIELlO, M. A.; SILVA, R. D. S.; CRUZ, M. A. B. D.; GUARIM NETO, G. Quintais urbanos de Mirassol D'OesteMT, Brasil: uma abordagem etnobotânica. Acta Amazônica, v. 40, n. 3, p. 451-470, 2010.
EICHEMBERG, M. T.; AMOROZO, M. C. M.; MOURA, L. C. Species composition and plant use in old urban homegardens in Rio Claro, Southeast of Brazil. Acta Botânica Brasílica, v. 23, n. 4, p. 1057-1075, 2009.

FLORENTINO, A. T. N.; ARAUJO, E. L.; ALBUQUERQUE, U. P. Contribuição de quintais agroflorestais na conservação de plantas da Caatinga, município de Caruaru, PE, Brasil. Acta Botânica Brasílica, v. 21, n. 1, p. 37-47, 2007.

FREITAS, A. V. L.; COELHO, M. F. B.; MAIA, S. S. S.; AZEVEDO, R. A. B. Plantas medicinais: um estudo etnobotânico nos quintais do Sítio Cruz, São Miguel, Rio Grande do Norte, Brasil. Revista Brasileira de Biociências, v. 10, n. 1, p. 48-59, 2012.

FREITAS A. V. L.; COELHO M. F. B.; PEREIRA Y. B.; FREITAS NETO E. C.; AZEVEDO R. A. B. Diversidade e usos de plantas medicinais nos quintais da comunidade de São João da Várzea em Mossoró, RN. Revista Brasileira de Plantas Medicinais. v. 17, n. 4 (Suppl 2), p. 845-856, 2015.

GAO, J.; HE, T.; LI, Q. M. Traditional home-garden conserving genetic diversity: a case study of Acacia pennata in southwest China. Conservation Genetics, v. 13, n. 4, p. 891-898, 2012.

GARCIA, B. N. R.; VIEIRA, T. A.; OLIVEIRA, F. A. Quintais agroflorestais e segurança alimentar em uma comunidade rural na Amazônia Oriental. Revista da Faculdade de Agronomia de La Plata. v. 114, n. 1, p. 67-73, 2015.

GERVAZIO, W. Agrobiodiversidade e qualidade do solo em quintais agroflorestais urbanos na cidade de Alta Floresta-MT. 2015. 136f. Dissertação (Programa de Pós-Graduação em Biodiversidade e Agroecossistemas Amazônicos) Universidade do Estado de Mato Grosso, Alta Floresta-MT. 2015.

GIRALDI, M.; HANAZAKI, N. Uso e conhecimento tradicional de plantas medicinais no Sertão do Ribeirão, Florianópolis, SC, Brasil. Acta Botânica Brasílica, v. 24, n. 2, p. 395-406, 2010.

GUARIM NETO, G.; AMARAL, C. N. Aspectos etnobotânicos de quintais tradicionais dos moradores de Rosário Oeste, Mato Grosso, Brasil. Polibotânica, n. 29, p. 191-212, 2010.

GUARIM NETO, G.; NOVAIS, A. M. Composição florística dos quintais da cidade de Castanheira. In: GUARIM NETO, G.; CARNIELLO, M. A. (Orgs.). Quintais mato-grossenses: espaços de conservação e reprodução de saberes. Cáceres: EDUNEMAT, 2008, 203 p.

IBGE, Instituto Brasileiro de Geografia e Estatística. Censo Demográfico 2010. Disponível em: 〈http://www.ibge.org.br〉. Acesso em: 12 out. 2018. 
KUMAR, B. M.; NAIR, P. K. R. Tropical home gardens: A time tested example of sustainable agroforestry. Springer Science, Dordrecht, The Netherlands, 2006, 380p.

MOREIRA, R. P. M.; GUARIM NETO, G. A flora medicinal dos quintais de Tangará da Serra, Mato Grosso, Brasil. Biodiversidade, v. 14, n. 1, p. 63-83, 2015.

MOSCA, V. P.; LOIOLA, M. I. B. Uso popular de plantas medicinais no Rio Grande do Norte, nordeste do Brasil. Revista Caatinga, v. 22, p. 225-234, 2009.

OAKLEY, E. Quintais Domésticos: uma responsabilidade cultural. Agriculturas, v. 1, n. 1, p. 37-39, 2004.

PAUlinO, R. C.; HENRIQUES, G. P. S. A.; MOURA, O. N. S.; COELHO, M. F. B.; AZEVEDO, R. A. B. Medicinal plants at the Sítio do Gois, Apodi, Rio Grande do Norte State, Brazil. Revista Brasileira de Farmacognosia, v. 22, n. 1, p. 29-39, 2012.

PEREIRA, P. V. M.; FIGUEIREDO NETO, L. F. Conservação de espécies florestais: um estudo em quintais agroflorestais no município de Cáceres-MT. Revista Eletrônica em Gestão, Educação e Tecnologia Ambiental, v. 19, n. 3, p. 783-793, 2015.

PERNA, T. A.; LAMANO-FERREIRA, A. P. N. Revisão Bibliométrica Sobre o Cultivo de Plantas Medicinais em Quintais Urbanos em Diferentes Regiões do Brasil (2009-2012). UNOPAR Científica Ciências Biológicas e da Saúde, v. 16, n. 1, p. 61-67, 2014.
PILlA, M. A. C.; AMOROZO, M. C. M.; FURLAN, A. Obtenção e uso das plantas medicinais no distrito de Martim Francisco, Município de Mogi-Mirim, SP, Brasil. Acta Botânica Brasílica, v. 20, n. 4, p. 789-802, 2006.

SANTOS, A. S.; OLIVEIRA, L. C. L.; CURADO, F. F.; AMORIM, L. O. Caracterização e desenvolvimento de quintais produtivos agroecológicos na comunidade Mem de Sá, Itaporanga d'Ajuda-Sergipe. Revista Brasileira de Agroecologia. v. 8, n. 2, p. 100-111, 2013.

SANTOS, S. Um estudo etnoecológico dos quintais de Alta Floresta-MT. 2004. 166f. Dissertação (Mestrado em Ecologia e Conservação da Biodiversidade), Universidade Federal de Mato Grosso, Cuiabá, 2004.

SEMEDO, R. J. C. G.; BARBOSA, R. I. Árvores frutíferas nos quintais urbanos de Boa Vista, Roraima, Amazônia brasileira. Acta Amazônica, v. 37, n. 4, p. 497-504, 2007.

VALADÃO, L. M.; AMOROZO, M. C. M.; MOTTA, D. G. Produção de alimentos na unidade domiciliar, dieta e estado nutricional: a contribuição dos quintais em um assentamento rural no Estado de São Paulo. In: ALBUQUERQUE, U. P.; ALMEIDA, C. F. B. (Orgs.). Tópicos em Conservação e Etnobotânica de Plantas Alimentícias. Recife: NUPPEA, p. 92115, 2006.

WINKLERPRINS, A.; OLIVEIRA, P. S. S. Urban Agriculture in Santarém, Pará, Brazil: diversity and circulation of cultivated plants in urban homegardens. Boletim do Museu Paraense Emílio Goeldi. Ciências Humanas. v. 5, n. 3, p. 571-585, 2010. 